\title{
Exercise-induced changes in stress hormones and cell adhesion molecules in obese men
}

\author{
Jinkyung Park' \\ Darryn S Willoughby ${ }^{2}$ \\ Joon Jin Song ${ }^{3}$ \\ Brian C Leutholtz ${ }^{2}$ \\ Yunsuk Koh² \\ 'Department of Kinesiology, \\ George Mason University, Manassas, \\ VA, USA; ${ }^{2}$ Department of Health, \\ Human Performance, Recreation, \\ Baylor University, Waco, TX, USA; \\ ${ }^{3}$ Department of Statistical Science, \\ Baylor University, Waco, TX, USA
}

Correspondence: Yunsuk Koh Baylor University, One Bear Place \#97313, Waco, TX 76798-7313, USA

Tel +I 2547104002

Fax + I 2547103527

Email Yunsuk_Koh@baylor.edu
This article was published in the following Dove Press journal: Journal of Inflammation Research

\begin{abstract}
Purpose: The current study examined the relationship between exercise-induced changes in stress hormones (epinephrine, norepinephrine, and cortisol) and vascular inflammatory markers (soluble intracellular adhesion molecule-1 [sICAM-1], soluble endothelial selectin [sE-selectin], and soluble vascular adhesion molecule-1 [sVCAM-1]) in obese men over a 24-hour period following exercise at lower and higher intensity.
\end{abstract}

Patients and methods: Fifteen physically inactive, obese, college-aged men performed a single bout of cycling exercise at lower and higher intensities (lower intensity: 50\% of maximal heart rate, and higher intensity: $80 \%$ of maximal heart rate) in random order. Overnight fasting blood samples were collected at baseline, immediately postexercise (IPE), 1-hour PE (1-h PE), and 24-hour PE. Changes in stress hormones and inflammatory markers were analyzed with a repeated-measures analysis of variance using Bonferroni multiple comparisons and a linear regression analysis $(p<0.05)$.

Results: sICAM-1, sVCAM-1, epinephrine, and norepinephrine did not change over time, while sE-selectin was significantly lower at $1-\mathrm{h}$ PE $(10.25 \pm 1.07 \mathrm{ng} / \mathrm{mL}, p=0.04)$ than at baseline $(12.22 \pm 1.39 \mathrm{ng} / \mathrm{mL})$. Cortisol and sICAM-1 were negatively related at 1 -h PE following lowerintensity exercise $\left(r^{2}=0.34, p=0.02\right)$, whereas cortisol and sVCAM- 1 were positively related at IPE following higher-intensity exercise $\left(r^{2}=0.36, p=0.02\right)$.

Conclusion: Regardless of intensity, an acute bout of aerobic exercise may lower sE-selectin in sedentary obese men. Responses of cortisol are dependent on exercise intensity, and cortisol may be a key stress hormone playing a major role in regulating sICAM-1 and sVCAM-1.

Keywords: vascular inflammation, ICAM-1, VACM-1, E-selectin, stress hormones, exercise intensity

\section{Introduction}

Atherosclerosis is the most common type of cardiovascular disease (CVD) ${ }^{1}$ and is strongly associated with elevated vascular inflammatory markers, such as cell adhesion molecules (CAMs). CAMs are glycoproteins expressed on the surface of various cells and play a crucial role in many cellular processes. ${ }^{2}$ In particular, intracellular adhesion molecules-1 (ICAM-1), vascular cell adhesion molecules-1 (VCAM-1), and E-selectin are considered the key molecules that are responsible for transmigrating leukocytes into the vascular intima, which in turn initiates the inflammatory process. ${ }^{3}$ However, a direct measurement of the actual expression of CAMs on endothelia is quite difficult in humans, whereas a soluble form of CAMs in the circulation, known 
as soluble CAMs (sCAMs), can be easily obtained from blood samples and indirectly reflects the rate of endothelial expression of CAMs. ${ }^{4}$

Stress hormones, such as catecholamines (epinephrine [E] and norepinephrine [NE]) and cortisol, can modulate the CAM metabolism. When catecholamine receptors on leukocytes are activated in response to physical activity or stress, catecholamines and cortisol stimulate the expression of CAMs, leading to an increase of sCAMs in circulation. ${ }^{5}$ Therefore, it is presumed that the magnitude of changes in catecholamines and cortisol induced by physical activity can lead to a change in sCAMs, which in turn may be dependent upon the intensity of exercise performed. ${ }^{6}$

Exercise can be of great benefit for preventing CVD and possibly provide a positive impact on vascular health. However, there is controversy regarding the effects of different intensities of exercise on the responses of CAMs. ${ }^{7-12}$ Despite substantial evidence suggesting that exercise may influence CAMs, ${ }^{7-12}$ it is still unclear how exercise at different intensities (lower vs higher) and exercise-induced stress hormones affect CAMs. Moreover, the majority of the previous studies focused on investigating the effect of exercise on CAMs in recreational athletes and trained individuals $^{10,11,13-22}$ rather than in sedentary overweight or obese individuals. In obesity, excessive adipose tissue produces a number of inflammatory cytokines that contribute to acute and chronic inflammation. In addition, the major risk factors for CVD include male sex, physical inactivity, and obesity. ${ }^{23}$ Therefore, the purpose of the current study was to examine the response of sCAMs (sICAM-1, sVCAM-1, and sE-selectin) in sedentary obese men following exercise performed at different intensities and to determine whether there was any relationship between exercise-induced stress hormones and sCAMs.

\section{Patients and methods}

\section{Participants}

Fifteen physically inactive obese men (body mass index $\geq 30 \mathrm{~kg} / \mathrm{m}^{2}$ ) between the ages of 18 and 30 years, who had not engaged in any form of physical activity ( $<2$ days per week) for at least 6 months prior to the study, volunteered for this study. All participants were at low risk for developing CVD and had no contraindications to exercise as outlined by the American College of Sports Medicine. The study protocol was reviewed and approved by the Baylor University Institutional Review Board for human subjects, and all participants signed written informed consent documents prior to performing any study protocols.

\section{Maximal heart rate test protocol}

The maximal heart rate (HRmax) test was conducted using a Monark cycle ergometer (Medgraphics, Ergomedic, Model No. 828E; Monark, St Paul, MN, USA) based on the protocol used by Keytel et al ${ }^{24}$ to estimate the participants' HRmax, which was defined as maximal heart rate achieved at the point of maximal exhaustion. During the HRmax test, the participants warmed up on the bicycle for 2 minutes with a workload of $2 \mathrm{~W} / \mathrm{kg}$ of body weight. After the warm-up, the participants began cycling at 70 revolutions per minute (rpm) with an exercise intensity of $3.33 \mathrm{~W} / \mathrm{kg}$ of body weight for 150 seconds (stage 1). Upon completion of stage 1, the workload increased by an additional $50 \mathrm{~W}$ for another 150 seconds (stage 2). Thereafter, the workload increased by $25 \mathrm{~W}$ every 150 seconds until the participants could no longer maintain $70 \mathrm{rpm}$ or volitionally stopped the test. The participants wore a heart rate monitor (Polar Electro Inc., Lake Success, NY, USA) around the chest and heart rate was recorded every minute throughout the test.

\section{Study design and exercise trials}

In a randomized, crossover design, each participant performed two exercise trials consisting of the lower- $(50 \%$ of HRmax) and higher-intensity exercise ( $80 \%$ of HRmax) trials. Each exercise trial was randomly assigned and performed at least 7 days apart to allow the participants to fully recover and return to basal conditions. During each exercise trial, the participants exercised on the cycle ergometer until they expended $300 \mathrm{kcal}$, which was calculated using the following equation by Keytel et $\mathrm{al}^{24}$ [gender $\times(-55.0969+0.6309$ $\times$ heart rate $+0.1988 \times$ body weight $(\mathrm{kg})+0.2017 \times$ age $)$ $+(1-$ gender $) \times(-20.4022+0.4472 \times$ heart rate $-0.1263 \times$ body weight $(\mathrm{kg})+0.074 \times$ age $)$; where gender $=1$ for males and 0 for females]. The participants refrained from any types of physical activity until completing the last blood draw at 24-hour following each exercise trial.

\section{Blood sample collection}

Overnight fasting blood samples were collected at baseline (PRE), immediately postexercise (IPE), 1-hour PE (1-h PE), and 24-hour PE (24-h PE) for each exercise trial. After 10 minutes of resting in a chair, venous blood from the antecubital vein was collected into a serum separator tube. Immediately after blood draws, blood samples remained at room temperature for 20 minutes to be clotted and then were centrifuged $(1,000 \mathrm{~g})$ for 20 minutes to separate the serum. Aliquots of serum samples were pipetted into $1.5-\mathrm{mL}$ polypropylene tubes and immediately frozen at $-80^{\circ} \mathrm{C}$ for later analyses. 


\section{Analysis of sCAMs and stress hormones}

Serum samples were analyzed in duplicate for sICAM-1 and sVCAM-1 (Cat\# ZF000000AY; Bio-Rad Laboratories, Hercules, CA, USA) by a multiplex flow immunoassay. The concentrations of sICAM- 1 and SVCAM- 1 were determined by median fluorescent intensity using a five-parameter logistic curve-fit. Serum samples in duplicate were analyzed for sEselectin (Kit\# EK0501; BOSTER, Fermont, CA, USA) by an enzyme-linked immunosorbent assay. The optical density was read at $450 \mathrm{~nm}$ using a spectrophotometer (SmartSpec Plus; Bio-Rad Laboratories) and the concentration was determined by a semi-log fit. Serum samples were analyzed in duplicate for E and NE (Kit\# EA613/192; DLD Diagnostika GmbH, Adlerhost, Hamburg, Germany) and cortisol (Kit\# DKO001; DiaMetra, Milan, Italy) by enzyme-linked immunosorbent assay. The optical density was read at $450 \mathrm{~nm}$ by the spectrophotometer (SmartSpec Plus; Bio-Rad Laboratories) and the concentrations were determined by a four-parameter logistic fit. Each assay was performed as instructed by the manufacturer's assay procedure. Intra- and interassay $\% \mathrm{CV}$ for each marker was as follows: sICAM-1 $(5.91 \%$ and $7.25 \%)$, sVCAM-1 (6.32\% and 7.97\%), sE-selectin ( $4.82 \%$ and $4.54 \%)$, E ( $8.12 \%$ and $9.01 \%)$, NE (5.85\% and $6.67 \%)$, and cortisol (5.80\% and $4.05 \%)$.

\section{Statistical analyses}

All statistical analyses were performed using the IBM Statistical Package for the Social Sciences 23.0 (IBM SPSS, Armonk, NY, USA) and reported as a mean \pm standard error (SE) unless stated otherwise. The Shapiro-Wilk test with the normal Q-Q plots was used to examine normality, and all data were log-transformed if normality did not exist. Effect sizes were calculated for each outcome variable using Cohen's $d$. A $2 \times 4$ (intensity $\times$ time) factorial analysis of variance (ANOVA) with repeated measures was used to examine the effects of exercise intensity (lower vs. higher) on sCAMs (sICAM-1, sVCAM-1, sE-selectin) over the 24-hour period (PRE, IPE, 1-h PE, and 24-h PE). Since it has been well documented that exercise typically yields an acute increase in stress hormones during exercise, which in turn return to the pre-exercise condition within a couple of hours upon completion of exercise, ${ }^{25}$ the current study only included the blood samples of PRE, IPE, and 1-h PE to examine the responses of stress hormones (E, NE, and cortisol). A $2 \times 3$ (intensity $\times$ time) ANOVA with repeated measures was used to examine the responses of E, NE, and cortisol at PRE, IPE, and 1-h PE. If there were significant main effects on time, Bonferroni pairwise comparisons were conducted as post hoc tests to locate the significant mean differences. If a significant interaction between intensity and time was found, a follow-up simple effects test with Bonferroni pairwise comparisons was conducted as a post hoc test. Additionally, linear regression analysis was conducted to examine the relationship between sCAMs and stress hormones. A $p$-value $<0.05$ was set for the statistical significance.

\section{Results}

The anthropometric data of the participants are shown in Table 1. The average HR and duration for each lowerand higher-intensity exercise trial were $98.80 \pm 1.82 \mathrm{bpm}$ (42.00 \pm 1.77 minutes) and $154.20 \pm 2.76 \mathrm{bpm}(19.00 \pm 0.53$ minutes), respectively. One of the major findings of the present study was that either lower- or higher-intensity of aerobic exercise did not significantly change sICAM-1 or sVCAM-1 (Table 2 ), while sE-selectin was significantly $(p=0.04)$ reduced following exercise, regardless of intensity. The post hoc test indicated that sE-selectin at $1-\mathrm{h} \operatorname{PE}(10.25 \pm 1.07 \mathrm{ng} / \mathrm{mL})$ was significantly lower than PRE $(12.22 \pm 1.39 \mathrm{ng} / \mathrm{mL}, p=0.01)$ and IPE $(12.20 \pm 1.55 \mathrm{ng} / \mathrm{mL}, p=0.02)$ (Figure 1). There were no significant changes in $\mathrm{E}$ or NE, whereas cortisol had a significant interaction effect between exercise intensity and time (Table 3). The further analysis post hoc revealed that cortisol at IPE $(187.52 \pm 31.09 \mathrm{ng} / \mathrm{mL}, p=0.01)$ and at $1-\mathrm{h}$ PE $(156.24 \pm 31.11 \mathrm{ng} / \mathrm{mL}, p=0.01)$ were significantly lower than PRE (259.74 $\pm 23.07 \mathrm{ng} / \mathrm{mL})$, respectively, during the lower-intensity exercise trial. In addition, cortisol at 1-h PE $(189.35 \pm 31.11 \mathrm{ng} / \mathrm{mL})$ significantly decreased by $27.76 \%$ $(p=0.01)$ from IPE $(262.12 \pm 31.09 \mathrm{ng} / \mathrm{mL})$ following higherintensity exercise (Figure 2).

Table 4 displays the relationship between sCAMs and exercise-induced stress hormones following the lower- and

Table I Anthropometric data of the participants

\begin{tabular}{ll}
\hline Variables & Mean \pm SE \\
\hline Age $($ years $)$ & $21.73 \pm 0.47$ \\
Height $(\mathrm{cm})$ & $177.09 \pm 2.27$ \\
Bodyweight $(\mathrm{kg})$ & $107.88 \pm 4.83$ \\
BMI $\left(\mathrm{kg} / \mathrm{m}^{2}\right)$ & $34.25 \pm 1.17$ \\
Body fat $(\%)$ & $31.56 \pm 1.17$ \\
SBP $(\mathrm{mmHg})$ & $122.13 \pm 7.98$ \\
DBP $(\mathrm{mmHg})$ & $72.933 \pm 7.49$ \\
Lean mass $(\mathrm{kg})$ & $64.62 \pm 2.37$ \\
Fat mass $(\mathrm{kg})$ & $31.77 \pm 2.5$ \\
HRrest $(\mathrm{bpm})$ & $69.27 \pm 9.43$ \\
HRmax $(\mathrm{bpm})$ & $190.93 \pm 3.35$ \\
\hline
\end{tabular}

Abbreviations: SE, standard error; BMI, body mass index; SBP, systolic blood pressure; DBP, diastolic blood pressure; HRrest, resting heart rate; HRmax, maximal heart rate. 
Table 2 Changes in sCAMs over 24 hours

\begin{tabular}{llllll}
\hline Intensity & Variables & PRE & IPE & I-hour PE & 24-hour PE \\
\hline LI & sICAM-I $(\mathrm{ng} / \mathrm{mL})$ & $126.80 \pm 7.27$ & $120.43 \pm 8.86$ & $118.64 \pm 7.99$ & $126.17 \pm 8.25$ \\
& sVCAM-I $(\mathrm{ng} / \mathrm{mL})$ & $119.01 \pm 7.00$ & $112.25 \pm 8.25$ & $107.08 \pm 6.21$ & $116.19 \pm 5.83$ \\
& sE-selectin $(\mathrm{ng} / \mathrm{mL})$ & $12.99 \pm 8.85$ & $11.36 \pm 6.55$ & $9.60 \pm 4.81$ & $10.48 \pm 6.67$ \\
$\mathrm{HI}$ & sICAM-I $(\mathrm{ng} / \mathrm{mL})$ & $127.52 \pm 7.27$ & $137.97 \pm 8.86$ & $129.50 \pm 7.99$ & $127.84 \pm 8.25$ \\
& sVCAM-I $(\mathrm{ng} / \mathrm{mL})$ & $119.87 \pm 7.00$ & $130.87 \pm 8.25$ & $122.74 \pm 6.21$ & $121.34 \pm 5.83$ \\
& sE-selectin $(\mathrm{ng} / \mathrm{mL})$ & $11.45 \pm 6.13$ & $13.04 \pm 10.10$ & $10.90 \pm 6.76$ & $12.14 \pm 5.83$ \\
\hline
\end{tabular}

Note: All data are presented as mean \pm SE.

Abbreviations: sCAM, soluble cell adhesion molecule; LI, lower-intensity; HI, higher-intensity; sICAM-I, soluble intracellular adhesion molecule-I; sVCAM-I, soluble vascular cellular adhesion molecule-I; sE-selectin, soluble endothelial selectin; PRE, pre-exercise; IPE, immediately postexercise; PE, postexercise; SE, standard error.

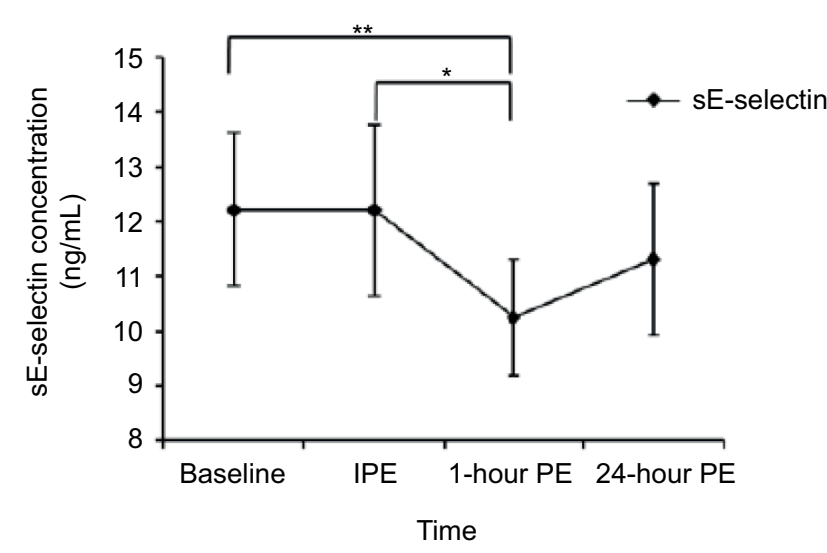

Figure I sE-selectin concentration at each time point.

Notes: *A significant difference between IPE and I-hour PE ( $p=0.029)$, **a significant difference between baseline and I-hour PE $(p=0.0 \mathrm{I})$.

Abbreviations: $s E-$ selectin, soluble endothelial selectin; IPE, immediately postexercise; PE, postexercise.

Table 3 Changes in stress hormones at each time point by exercise intensity

\begin{tabular}{lllll}
\hline Intensity & Variables & PRE & IPE & I-hour PE \\
\hline $\mathrm{LI}$ & $\mathrm{E}(\mathrm{pg} / \mathrm{mL})$ & $44.07 \pm 6.78$ & $41.19 \pm 9.06$ & $41.80 \pm 7.85$ \\
& $\mathrm{NE}(\mathrm{pg} / \mathrm{mL})$ & $257.97 \pm 34.99$ & $246.25 \pm 49.93$ & $299.87 \pm 26.46$ \\
& $\mathrm{COR}$ & $259.74 \pm 23.07$ & $187.52 \pm 31.09^{\mathrm{a}}$ & $156.24 \pm 31 . \mathrm{I}^{\mathrm{a}}$ \\
& $(\mathrm{ng} / \mathrm{mL})$ & & & \\
$\mathrm{HI}$ & $\mathrm{E}(\mathrm{pg} / \mathrm{mL})$ & $44.29 \pm 8.02$ & $48.35 \pm 10.72$ & $38.05 \pm 9.29$ \\
& $\mathrm{NE}(\mathrm{pg} / \mathrm{mL})$ & $214.71 \pm 41.40$ & $335.67 \pm 59.08$ & $257.39 \pm 31.31$ \\
& $\mathrm{COR}$ & $243.75 \pm 23.07$ & $262.12 \pm 31.09$ & $189.35 \pm 31.1 I^{\mathrm{b}}$ \\
& $(\mathrm{ng} / \mathrm{mL})$ & & & \\
\hline
\end{tabular}

Notes: All data are presented as mean \pm SE. aSignificantly different from PRE $(p=0.0 \mathrm{I})$, 'significantly different from IPE $(p=0.0 \mathrm{I})$.

Abbreviations: LI, lower-intensity; HI, higher-intensity; E, epinephrine; NE, norepinephrine; COR, cortisol; PRE, pre-exercise; IPE, immediately postexercise; $\mathrm{PE}$, postexercise; SE, standard error.

higher-intensity exercise trials. Cortisol and sICAM-1 had a weak, but significant, negative relationship $\left(r^{2}=0.34, p=0.02\right)$ at 1-h PE during the lower-intensity exercise trial, while cortisol and SVCAM- 1 had a positive relationship $\left(r^{2}=0.36\right.$, $p=0.02$ ) at IPE during the higher-intensity exercise trial. Cortisol and sE-selectin had a negative relationship at PRE during the lower- $\left(r^{2}=0.31, p=0.03\right)$ and higher-intensity $\left(r^{2}=0.70\right.$,

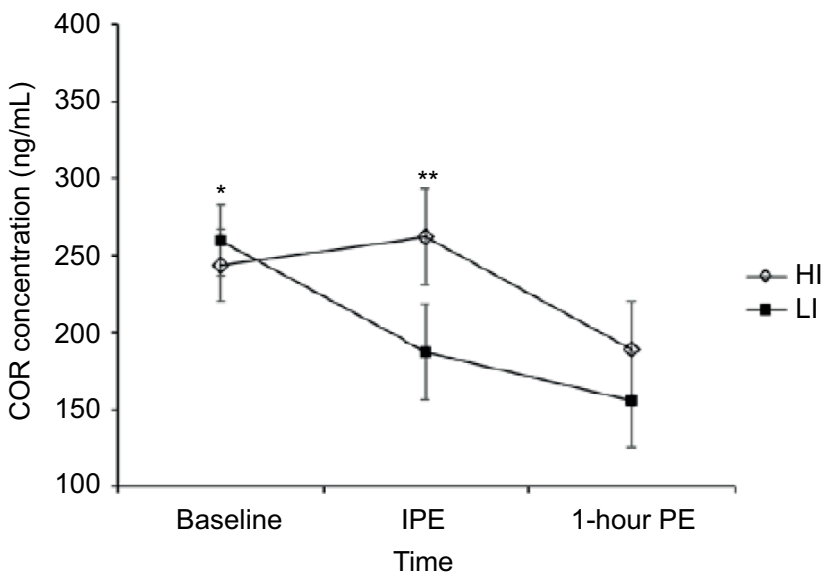

Figure $\mathbf{2}$ Changes in cortisol concentration at each time point following lower and higher intensities of exercise.

Notes: *Significantly different from IPE and I-hour PE for LI ( $p=0.009$ and 0.001 , respectively), **significantly different from I-hour $\mathrm{PE}$ for $\mathrm{HI}(p=0.00 \mathrm{I})$.

Abbreviations: IPE, immediately postexercise; PE, postexercise; HI, higherintensity; LI, lower-intensity.

$p=0.01)$ exercise trials. In addition, $\mathrm{E}$ and $\mathrm{sE}-\mathrm{selectin}$ had a positive relationship $(r=0.56, p=0.01)$ at $1-\mathrm{h}$ PE during the higher-intensity exercise trial.

\section{Discussion}

The present study examined the acute responses of sCAMs and their association with exercise-induced stress hormones in sedentary obese men following a single bout of aerobic exercise at two different intensities (lower: $50 \%$ and higher: $80 \%$ HRmax). In the current study, either lower- or higherintensity exercise did not significantly alter sICAM-1 and sVCAM-1. A similar result has been reported by several previous studies that utilized a variety of subject populations including physically active ${ }^{21}$ and sedentary ${ }^{17}$ men along with patients with type- 2 diabetes. ${ }^{16}$ Several studies, however, have reported an acute increase in SICAM-1 and SVCAM-1 immediately following exercise, which in turn returned to the pre-exercise levels within several hours postexercise. For instance, healthy men showed increased sICAM-1 by 
Table 4 Relationships between sCAMs and stress hormones

\begin{tabular}{|c|c|c|c|c|c|c|c|c|}
\hline \multirow[t]{2}{*}{ Intensity } & \multirow[t]{2}{*}{ Time } & \multirow[t]{2}{*}{ Predictor } & \multicolumn{2}{|c|}{ sICAM-I } & \multicolumn{2}{|c|}{ sVCAM-I } & \multicolumn{2}{|c|}{ sE-selectin } \\
\hline & & & $R^{2}$ (\%) & $p$-value & $R^{2}(\%)$ & $p$-value & $R^{2}(\%)$ & $p$-value \\
\hline \multirow[t]{9}{*}{ LI } & PRE & $\mathrm{E}$ & 0 & $\mathrm{I}$ & 0.3 & 0.86 & 0.1 & 0.89 \\
\hline & & $\mathrm{NE}$ & -11.8 & 0.21 & 5.2 & $0.4 \mathrm{I}$ & 2.3 & 0.59 \\
\hline & & COR & -1.6 & 0.65 & 7.6 & 0.32 & -31.5 & $0.03 *$ \\
\hline & IPE & $\mathrm{E}$ & 1.3 & 0.68 & 8.4 & 0.3 & 1.4 & 0.67 \\
\hline & & $\mathrm{NE}$ & 0 & 1 & 4.7 & 0.44 & 5.7 & 0.39 \\
\hline & & COR & -2.9 & 0.54 & 10.2 & 0.25 & -10.1 & 0.25 \\
\hline & I-hour PE & $\mathrm{E}$ & 6.4 & 0.39 & 7.3 & 0.35 & 11.8 & 0.22 \\
\hline & & NE & -1.9 & 0.64 & -0.5 & 0.81 & 19 & 0.12 \\
\hline & & COR & -34 & $0.02 *$ & -5.3 & $0.4 I$ & -7.4 & 0.33 \\
\hline \multirow[t]{9}{*}{$\mathrm{HI}$} & PRE & $\mathrm{E}$ & 2.8 & 0.57 & 3.2 & 0.54 & 0.3 & 0.85 \\
\hline & & $\mathrm{NE}$ & -0.6 & 0.79 & 2.7 & 0.57 & -1.3 & 0.69 \\
\hline & & COR & -5.7 & 0.39 & 11.4 & 0.22 & -69.7 & $0.01 *$ \\
\hline & IPE & $\mathrm{E}$ & -16.8 & 0.15 & -19.3 & 0.10 & 7.2 & 0.34 \\
\hline & & NE & -2.5 & 0.59 & -6.5 & 0.36 & 18.7 & 0.10 \\
\hline & & COR & 0 & 0.97 & 36 & $0.02 *$ & -13.3 & 0.18 \\
\hline & I-hour PE & $\mathrm{E}$ & -1.4 & 0.73 & -13.3 & 0.27 & 55.6 & $0.01 *$ \\
\hline & & NE & -0.2 & 0.90 & -19.5 & 0.17 & 19.1 & 0.18 \\
\hline & & COR & -.4 & 0.81 & 23.2 & 0.07 & -4.6 & 0.44 \\
\hline
\end{tabular}

Note: $* p<0.05$.

Abbreviations: sCAMs, soluble cell adhesion molecules; LI, lower-intensity; HI, higher-intensity; sICAM-I, soluble intracellular adhesion molecule-I; sVCAM-I, soluble vascular adhesion molecule-I; sE-selectin, soluble endothelial selectin; E, epinephrine; NE, norepinephrine; COR, cortisol; PRE, pre-exercise; IPE, immediately postexercise; $\mathrm{PE}$, postexercise.

$24 \%$ immediately following a maximal exercise test, which then returned to the pre-exercise level 1-h PE. ${ }^{20}$ Untrained healthy males demonstrated increased both sICAM-1 and sVCAM-1 by $10 \%$ immediately following maximal cycling exercise, whereas only a minor elevation (sICAM-1: 5\% and sVCAM-1: 1\%) was observed following moderate-intensity exercise ( $60 \%$ of maximal cycling exercise). ${ }^{17}$ Most of the studies including the current study that have reported no significant changes in sICAM-1 or sVCAM-1 utilized a moderate to submaximal exercise intensity protocol with relatively short duration, ${ }^{16,17,21}$ whereas it seems that maximalintensity exercise causes a transitory elevation of SICAM-1 and sVCAM-1. Thus, aerobic exercise at any intensity, except for maximal intensity, may not negatively influence sICAM-1 or sVCAM-1.

Only a few studies have previously examined the responses of sE-selectin to exercise and most of these studies used untrained nonobese men and reported no changes in sE-selectin. ${ }^{16,17,26}$ However, it should be noted that sedentary obese men in the current study significantly decreased sE-selectin following exercise, regardless of intensity. This result was similar to the study by Roberts and associates that reported a reduction in sE-selectin following moderateintensity aerobic exercise accompanied with a dietary intervention in overweight/obese male patients with type 2 diabetes. ${ }^{27}$ sE-selectin mediates the initial step of leukocyte transmigration and rolling, ${ }^{28}$ and inflammatory stimuli are known to induce maximum expression of E-selectin on the surface of endothelial cells. ${ }^{29}$ Thus, it seems that aerobic exercise in obese individuals may improve endothelial health by lowering sE-selectin, which may further downregulate the activity of leukocyte transmigration and rolling.

The current literature is generally agreed that stress including physical exercise stimulates a secretion of stress hormones, such as catecholamines and cortisol, which could also influence sCAMs. ${ }^{7}$ However, we found no significant changes in catecholamines following either lower- or highintensity exercise. A possible explanation for the lack of changes in catecholamines in response to exercise may be related to our participants' characteristic - obese men. Indeed, obese individuals had significantly lower responses of catecholamines than the nonobese, both at rest and during exercise, possibly due to obesity-mediated decreases in adrenergic receptor sensitivity. ${ }^{30}$ Additionally, the current study observed a positive relation between $\mathrm{E}$ and $\mathrm{sE}$-selectin at 1-h PE during higher-intensity exercise. This result may be associated with exercise-induced beta-adrenergic stimulation since it has been reported that the expression of sE-selectin is mainly related to a response of $E$ to exercise. It has been reported that exercise may increase the recruitment of 
leukocytes through beta-adrenergic stimulation that interacts with $\mathrm{E}$ in endothelial tissue and induces an increase in the expression of E-selectin. ${ }^{7}$ One previous study also demonstrated that beta-adrenergic stimulation was required to upregulate sE-selectin, suggesting that $\mathrm{E}$ is directly related to changes in sE-selectin. ${ }^{31}$

Conversely, cortisol was significantly decreased at IPE and 1-h PE from baseline during lower-intensity exercise, while cortisol at IPE was significantly higher than at 1-h PE during higher-intensity exercise. This result was similar to the previous study, which suggested that an increase in cortisol following a brief exercise was influenced by the intensity of exercise, since exercise intensity above $60 \% \mathrm{VO}_{2} \max$ increases cortisol, while exercising below $50 \% \mathrm{VO}_{2} \max$ seems to reduce cortisol mainly due to an enhanced elimination and suppressed secretion of cortisol. ${ }^{32}$

We found a statistically significant relationship between sCAMs and cortisol. However, only sVCAM-1 at IPE showed a positive relationship with cortisol at IPE during higher-intensity exercise. An exercise-induced increase in cortisol during higher-intensity exercise may be related to an increase in proinflammatory cytokines, which consequently increases in sVCAM-1,33 while, interestingly, anti-inflammatory cytokines modulate only sVCAM-1. ${ }^{34}$ Thus, the increase in cortisol immediately following higherintensity exercise may cause an increase in sVCAM-1, primarily due to an exercise-induced increase in proinflammatory cytokines. Additionally, cortisol has an inhibitory effect on sICAM-1 by switching off the activated ICAM-1 gene through glucocorticoid receptors, and a low level of cortisol is also related to a decrease in inflammatory gene transcription. ${ }^{35}$ Thus, a low level of cortisol for a certain condition, such as low-intensity exercise, may suppress sICAM-1, and this may explain our result showing a negative relationship between cortisol and sICAM-1 at 1-h PE during lower-intensity exercise. One study has proposed a concentration-dependent, biphasic (both stimulatory and suppressive) effect of cortisol on inflammation. Effects of cortisol on a stimulus-induced inflammatory response can be either suppressive or stimulatory in a concentration- and time-dependent manner. ${ }^{36}$ Thus, our current results showing a positive relation with SVCAM-1 at IPE following higherintensity exercise and the negative relation with sICAM-1 at 1-h PE following lower-intensity suggest that exerciseinduced cortisol may have a biphasic effect on vascular inflammation and may play a central role in the regulation of sICAM-1 and sVCAM-1.

\section{Conclusion}

The findings of the current study suggest that an acute bout of exercise, regardless of intensity, lowers sE-selectin in sedentary obese men. It seems that higher volume and duration of exercise training may be necessary to significantly change both sICAM-1 and SVCAM-1. One of the important findings of the current study is that cortisol may be a key stress hormone playing an important role in regulating sICAM-1 and sVCAM-1. Future studies examining the mechanisms among pro- and anti-inflammatory cytokines, exercise-induced stress hormones, and CAMs are recommended to better understand the role of exercise in endothelial function.

\section{Acknowledgment}

The current study was funded by the Baylor University Research Committee Grant (number 330330291).

\section{Disclosure}

The authors report no conflicts of interest in this work.

\section{References}

1. Madamanchi NR, Vendrov A, Runge MS. Oxidative stress and vascular disease. Arterioscler Thromb Vasc Biol. 2005;25(1):29-38.

2. Galkina E, Ley K. Leukocyte influx in atherosclerosis. Curr Drug Targets. 2007;8(12):1239-1248.

3. Demerath E, Towne B, Blangero J, Siervogel RM. The relationship of soluble ICAM-1, VCAM-1, P-selectin and E-selectin to cardiovascular disease risk factors in healthy men and women. Ann Hum Biol. 2001;28(6):664-678.

4. Abe Y, El-Masri B, Kimball KT, et al. Soluble cell adhesion molecules in hypertriglyceridemia and potential significance on monocyte adhesion. Arterioscler Thromb Vasc Biol. 1998;18(5):723-731.

5. Black PH, Garbutt LD. Stress, inflammation and cardiovascular disease. J Psychosom Res. 2002;52(1):1-23.

6. Blake GJ, Ridker PM. Inflammatory bio-markers and cardiovascular risk prediction. J Intern Med. 2002;252(4):283-294.

7. Shephard RJ. Adhesion molecules, catecholamines and leucocyte redistribution during and following exercise. Sports Med. 2003;33(4):261-284.

8. Gleeson M. Immune function in sport and exercise. J Appl Physiol. 2007;103(2):693-699.

9. Verde TJ, Thomas SG, Moore RW, Shek P, Shephard RJ. Immune responses and increased training of the elite athlete. J Appl Physiol (1985). 1992;73(4):1494-1499.

10. Miles MP, Leach SK, Kraemer WJ, Dohi K, Bush JA, Mastro AM. Leukocyte adhesion molecule expression during intense resistance exercise. J Appl Physiol (1985). 1998;84(5):1604-1609.

11. Smith LL, Anwar A, Fragen M, Rananto C, Johnson R, Holbert D. Cytokines and cell adhesion molecules associated with high-intensity eccentric exercise. Eur J Appl Physiol. 2000;82(1-2):61-67.

12. Petridou A, Chatzinikolaou A, Fatouros I, et al. Resistance exercise does not affect the serum concentrations of cell adhesion molecules. Br J Sports Med. 2007;41(2):76.

13. Perez CJ, Nemet D, Mills PJ, Scheet TP, Ziegler MG, Cooper DM. Effects of laboratory versus field exercise on leukocyte subsets and cell adhesion molecule expression in children. Eur J Appl Physiol. 2001;86(1): 34-39. 
14. Akimoto T, Furudate M, Saitoh M, et al. Increased plasma concentrations of intercellular adhesion molecule-1 after strenuous exercise associated with muscle damage. Eur J Appl Physiol. 2002;86(3):185-190.

15. Simpson RJ, Florida-James GD, Whyte GP, Guy K. The effects of intensive, moderate and downhill treadmill running on human blood lymphocytes expressing the adhesion/activation molecules CD54 (ICAM-1), CD18 (beta2 integrin) and CD53. Eur J Appl Physiol. 2006;97(1):109-121.

16. Wang JS, Chen YW, Chow SE, Ou HC, Sheu WH. Exercise paradoxically modulates oxidized low density lipoprotein-induced adhesion molecules expression and trans-endothelial migration of monocyte in men. Thromb Haemost. 2005;94(4):846-852.

17. Jilma B, Eichler HG, Stohlawetz P, et al. Effects of exercise on circulating vascular adhesion molecules in healthy men. Immunobiology. 1997;197(5):505-512.

18. Goebel MU, Mills PJ. Acute psychological stress and exercise and changes in peripheral leukocyte adhesion molecule expression and density. Psychosom Med. 2000;62(5):664-670.

19. Li N, Wallen NH, Hjemdahl P. Evidence for prothrombotic effects of exercise and limited protection by aspirin. Circulation. 1999; 100(13):1374-1379.

20. Rehman J, Mills PJ, Carter SM, Chou J, Thomas J, Maisel AS. Dynamic exercise leads to an increase in circulating ICAM-1: further evidence for adrenergic modulation of cell adhesion. Brain Behav Immun. 1997; 11(4):343-351.

21. Gabriel B, Ratkevicius A, Gray P, Frenneaux MP, Gray SR. Highintensity exercise attenuates postprandial lipaemia and markers of oxidative stress. Clin Sci. 2012;123(5):313-321.

22. Nemet D, Hong S, Mills PJ, Ziegler MG, Hill M, Cooper DM. Systemic vs. local cytokine and leukocyte responses to unilateral wrist flexion exercise. J Appl Physiol (Bethesda, MD: 1985). 2002;93(2): 546-554.

23. Mendis S, Puska P, Norrving B. Global Atlas on Cardiovascular Disease Prevention and Control. Geneva: World Health Organization; 2011:14
24. Keytel LR, Goedecke JH, Noakes TD, et al. Prediction of energy expenditure from heart rate monitoring during submaximal exercise. $J$ Sports Sci. 2005;23(3):289-297.

25. Steinberg JG, Ba A, Bregeon F, Delliaux S, Jammes Y. Cytokine and oxidative responses to maximal cycling exercise in sedentary subjects. Med Sci Sports Exerc. 2007;39(6):964-968.

26. Brevetti G, De Caterina M, Martone VD, et al. Exercise increases soluble adhesion molecules ICAM-1 and VCAM-1 in patients with intermittent claudication. Clin Hemorheol Microcirc. 2001;24(3):193-199.

27. Roberts CK, Won D, Pruthi S, Lin SS, Barnard RJ. Effect of a diet and exercise intervention on oxidative stress, inflammation and monocyte adhesion in diabetic men. Diabetes Res Clin Pract. 2006;73(3):249-259.

28. Bevilacqua MP, Nelson RM, Mannori G, Cecconi O. Endothelialleukocyte adhesion molecules in human disease. Ann Rev Med. 1994;45:361-378.

29. Lasky LA. Selectins: interpreters of cell-specific carbohydrate information during inflammation. Science. 1992;258(5084):964-969.

30. Zouhal H, Lemoine-Morel S, Mathieu ME, Casazza GA, Jabbour G. Catecholamines and obesity: effects of exercise and training. Sports Med. 2013;43(7):591-600.

31. Flach RJ, Matevossian A, Akie TE, Negrin KA, Paul MT, Czech MP. $\beta 3$-Adrenergic receptor stimulation induces e-selectin-mediated adipose tissue inflammation. J Biol Chem. 2013;288(4):2882-2892.

32. Shephard RJ. Endocrinology of Physical Activity and Sport. 2nd ed. Vol 38. Ottawa, ON: NRC Research Press; 2013:122.

33. Mastorakos G, Pavlatou M, Diamanti-Kandarakis E, Chrousos GP. Exercise and the stress system. Hormones (Athens, Greece). 2005;4(2):73-89.

34. Hosokawa Y, Hosokawa I, Ozaki K, Nakae H, Matsuo T. Cytokines differentially regulate ICAM-1 and VCAM-1 expression on human gingival fibroblasts. Clin Exp Immunol. 2006;144(3):494-502.

35. Barnes PJ, Adcock IM. How do corticosteroids work in asthma? Ann Intern Med. 2003;139(5 Pt 1):359-370.

36. Yeager MP, Pioli PA, Guyre PM. Cortisol exerts bi-phasic regulation of inflammation in humans. Dose Response. 2010;9(3):332-347.
Journal of Inflammation Research

\section{Publish your work in this journal}

The Journal of Inflammation Research is an international, peer-reviewed open access journal that welcomes laboratory and clinical findings on the molecular basis, cell biology and pharmacology of inflammation including original research, reviews, symposium reports, hypothesis formation and commentaries on: acute/chronic inflammation; mediators of inflammation; cellular processes; molecular mechanisms; pharmacology and novel anti-inflammatory drugs; clinical conditions involving inflammation. The manuscript management system is completely online and includes a very quick and fair peer-review system. Visit http://www.dove press.com/testimonials.php to read real quotes from published authors. 